\title{
Study on Environmental Monitoring System Based on Internet of Animal Husbandry Things
}

\author{
Hengqin Zhao, ${ }^{1,}$, Jianzhai $\mathrm{Wu}^{2,3}$, Jianhua Zhang ${ }^{2,3}$, Jiajia $\mathrm{Liu}^{2,3}$, \\ Pu Zhao ${ }^{2,3}$, and Shuqing $\operatorname{Han}^{2,3, a}$ \\ ${ }^{1}$ School of the Earth Sciences and Resources, China University of Geosciencesロ\%HNDJ, \\ Beijing 100083, China \\ ${ }^{2}$ Agricultural Information Institute of Chinese Academy of Agricultural Sciences, Beijing, 100081, \\ China \\ ${ }^{3}$ Key Laboratory of Agri-information Services Technology, Ministry of Agriculture, Beijing, 100081, \\ China \\ ${ }^{4}$ ZhongxinJhua Certified Public Accountants LLP, Beijing 100037, China \\ ${ }^{a}$ Corresponding author, email: hanshuqing@caas.cn
}

Keywords: Environmental Parameters; IoT (internet of things); GPRS; Animal Husbandary

\begin{abstract}
In view of actual requirements for livestock and poultry breeding environment monitoring, an environmental monitoring system based on Internet of Animal Husbandry Things (IoAHT) was introduced in this paper. The environmental monitoring system includes environmental monitoring station, information management system, and mobile APP. The system has achieved the real-time acquisition, transmission, storage and view of breeding environment information. Users can view the current environment data in real-time through mobile APP. The system has advantages such as high measurement accuracy, low cost and convenient operation.
\end{abstract}

\section{Introduction}

Internet of Animal Husbandry Things (IoAHT) means to apply the internet of things (IoT) technology into animal husbandry production, business, management and service. By using breeding environment monitoring sensor, physiological sign monitoring sensor, audio information acquisition sensor, video information acquisition sensor and other perception equipments to collect animal growth environment information in ranch and individual sign information, it would obtain a lot of ranch information to carry out fusion and processing through wireless sensor network, mobile telecommunication network, Internet and other modern information transmission methods to realize process monitoring, scientific decision-making, and real-time service of pre-production, in-production and post-production in ranch through intelligent operation terminals so as to achieve the integration Internet of man, machine and material in ranch and further realize the goal of high yield, excellent quality, high efficiency, ecology and safety in animal husbandry and breeding [1]. Among them, the breeding environment monitoring is the first step to realize an IoAHT [2]. The research on planting environment monitoring system of farmland [3] and facility agriculture [4] has been carried out earlier. But the research and report on animal husbandry environment monitoring system were less. The change of breeding environment directly affects growth rate and production of livestock, especially harmful gases [5] [6]. For example, ammonia can cause a decline in animal health, and reduce the weight increment rate. In severe cases, it will lead to paralysis of central nervous system, myocardial injury and others. Hydrogen sulfide will affect the health of animals and poultry, and destroy the respiration system of animals [7]. Therefore, the research and development of environmental monitoring system of IoAHT is very important according to the actual monitoring requirements of livestock and poultry breeding environment. 


\section{Overall System Design}

The environmental monitoring system based on IoAHT is composed of three sections such as environmental monitoring station of IoAHT, information management system of IoAHT, and mobile APP of IoAHT. The environmental monitoring station of IoAHT can collect real-time information of breeding environment (temperature, humidity, light, and wind direction) and gas concentration (methane, ammonia, carbon dioxide and hydrogen sulfide) and other parameter information, and upload sensing data through wireless data transmission unit (DTU) to the information management system. The information management system is a platform to collect, organize, visualize and display the environmental monitoring data of IoAHT uploaded from the environmental monitoring stations. Farmers utilize mobile APP of IoAHT to access the server of IoAHT through WiFi or GPRS communication network so as to view the breeding environment information of IoAHT station in real-time. The structural system diagram was shown in Fig. 1.

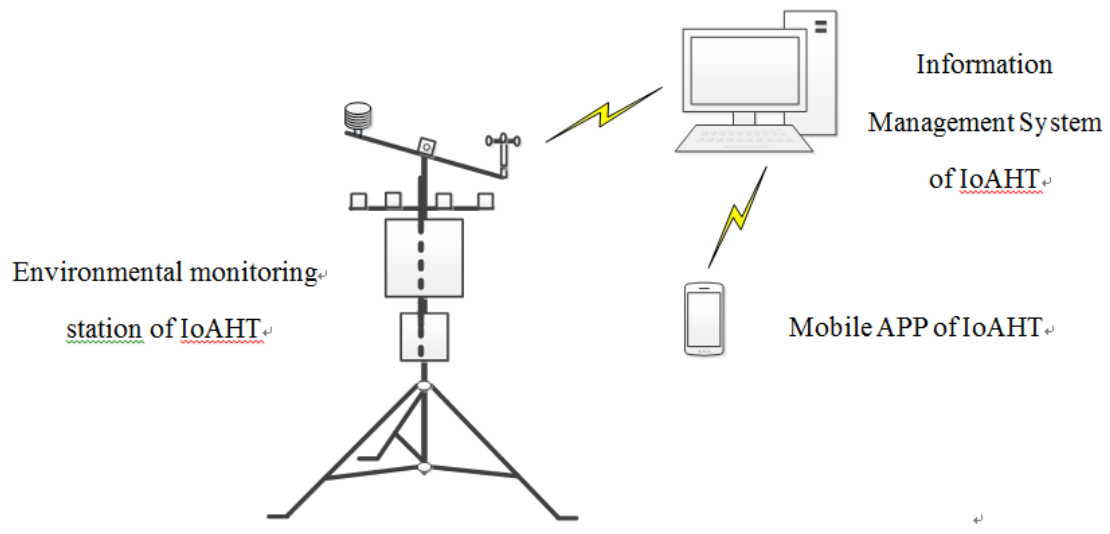

Fig.1. Environmental Monitoring System of IoAHT.

\section{Environmental Monitoring Station of IoAHT}

Environmental monitoring station of IoAHT includes controller, sensor node, solar power module, storage battery and stand. The controller is the core of environmental monitoring station of IoAHT. The controller reads environmental information collected by sensor node, and uploads data to servers. The structural diagram of environmental monitoring station was shown in Fig. 2. Based on the requirements for environmental monitoring, currently installed sensor nodes mainly include temperature sensor, light sensor, wind speed sensor, methane sensor, ammonia sensor, carbon dioxide sensor, and hydrogen sulfide sensor. In order to meet power supply requirements for field operations of monitoring station, the monitoring station has been equipped with solar power supply system to realize independent operations with uninterrupted power supply and get rid of the restriction of power supply conditions. Stand is composed of tripod, vertical pole and cross bar. Sensor nodes are fixed respectively on cross bars. Solar power module, controller and battery are fixed on vertical pole, as shown in Fig. 2. 


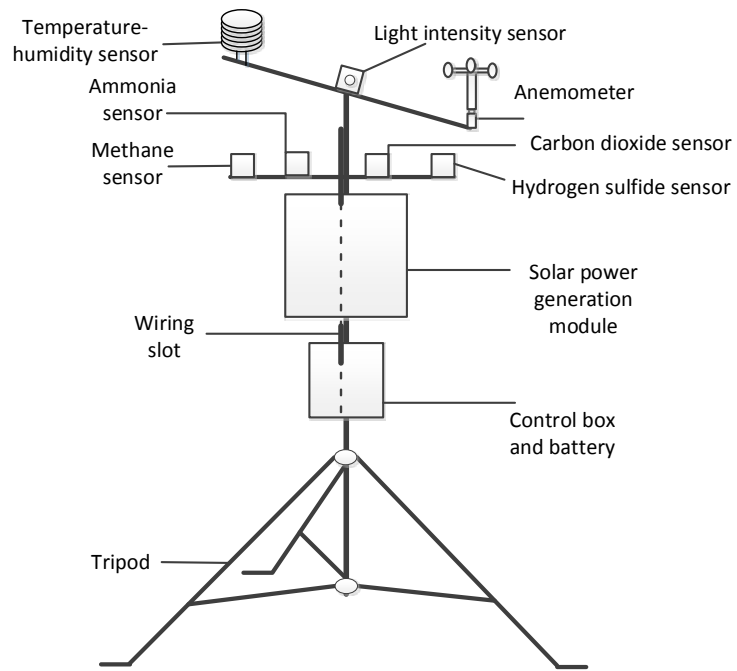

Fig.2. Structural Diagram on Monitoring Station of IoAHT.

The controller consists of a single chip micro-controller, power module, LCD display module, DTU, and RS485 interface chip. Among them, power module is connected with solar power supply system, and RS485 interface chip is connected with each sensor node. The hardware block diagram of controller was shown in Fig. 3. Micro-controller is the core of the system. It is used to communicate with RS-485 interface sensor node, read sensor data, display sensor data on LCD display module, and send sensor data to server through DTU. Functions of the micro-controller are relatively simple. Taking into account that requirements for cost reduction and low power consumption of monitoring station in the field work, STC12LE5A60S2 SCM has been selected for monitoring station. STC12LE5A60S2, which has high speed, low power consumption, anti-jamming and other characteristics, is manufactured by STCMICRO. The operating voltage of SCM is 2.2 3.6 V. The space of user application space is $60 \mathrm{~K}$. There are two serial ports that can meet the requirements for SCM to communicate with DTU data transmission module, LCD display module or sensor nodes at the same time.

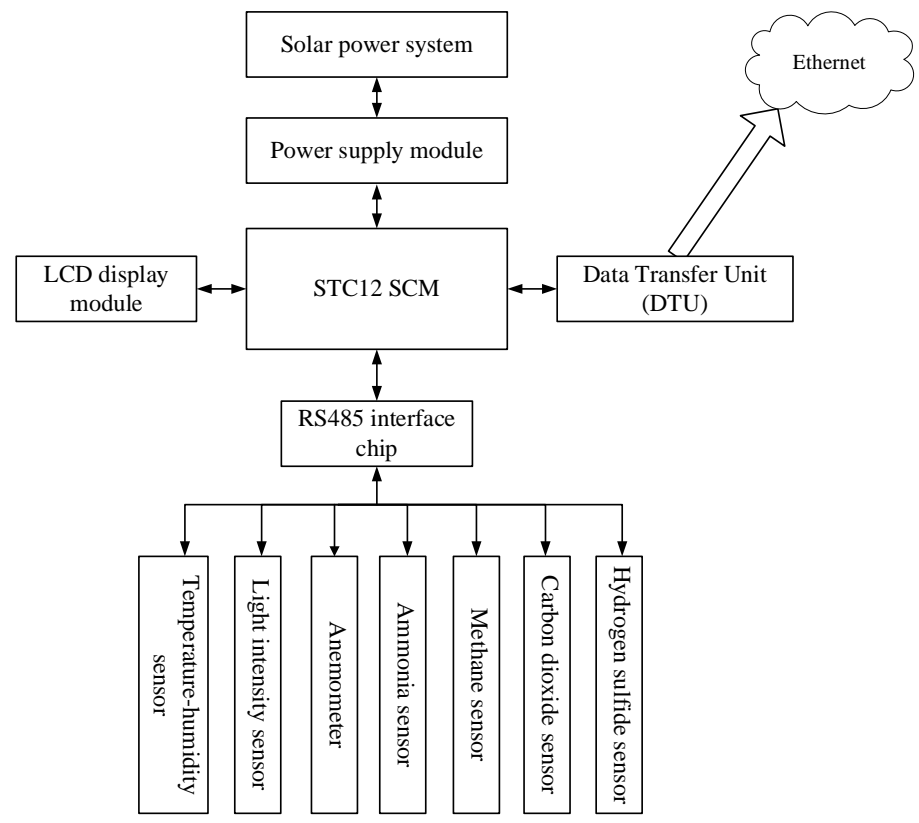

Fig.3. Hardware Block Diagram on Environmental Monitoring Station of IoAHT.

STC12 SCM and RS-485 interface chip SP3485 need a power supply of 3.3V. LCD display module needs a power supply of $5 \mathrm{~V}$. DTU and sensor nodes need a power supply of $12 \mathrm{~V}$. Solar-charging storage battery can provide $12 \mathrm{~V}$ power supply for DTU data transmission module and sensor nodes. $12 \mathrm{~V}$ power supply will be changed to $5 \mathrm{~V}$ power supply through DC/DC reduction voltage module composed of XL1509 to supply for LCD display module. 5V power supply will be 
changed to 3.3V by HT7533 linear regulator chips to supply STC12 SCM and RS-485 interface chip respectively.

Main system functions can be realized through serial communications. STC12 SCM includes two serial ports such as Serial Port 1 and Serial Port 2. Pins TXD and RXD of Serial Port 1 are connected respectively with UTXD1 and URXD1 of DTU. GPRS data transmission module is selected as DTU, which model is USR-GM1. With built in TCP/IP protocol stack, USR-GM1 can be set up and used easily. The module can be set to work in keepalive mode to prevent the wireless connection from being broken. Pins TXD2 and RXD2 of Serial Port 2 are connected with communication pin RXD_P and TXD_P of LCD display module. An industrial serial LCD touch screen is selected as LCD display module that the model is DMT48270M043. The module has advantages such as simple development, low power consumption and automatic backlight standup. Pins TXD2 and RXD2 of Serial Port 2 are connected with RS-485 interface chip SP3485 at the same time. SP3485 is a half-duplex transceiver with low power consumption to meet the requirements of RS-485 serial protocol, convert TTL level of STC12 SCM into RS-485 level, and realize the acquisition of sensing data. Sensor node is vulnerable to severe damages of natural environment such as hot sun, heavy rain, high temperature, high humidity, dew, frost, ice, haze, dust and so on. At the same time, disconnections of electrical connections caused by small animals and damages of sensor probe have occurred sometimes. In order to solve the problems, broken sensor nodes need to be replaced and updated periodically. All selected sensor node shall support the standard MODBUS communication protocol. The measuring range of each module is shown as follows: temperature measuring range: $-40 \sim 120^{\circ} \mathrm{C}$; humidity measuring range: $0 \sim 100 \% \mathrm{RH}$. Light intensity measuring range: $0 \sim 200,000 \mathrm{LUX}$. Wind speed measuring range: $0 \sim 30 \mathrm{~m} / \mathrm{s}$. Ammonia measuring range: 0 100 PPM. Carbon dioxide measuring range: 0 5,000 PPM. Hydrogen sulfide measuring range: $0 \sim 100$ PPM. Methane measuring range: $0 \sim 100 \%$ LEL.

First, initialize SCM ports. Set the parameters of Serial Port 1, and set the baud rate to 115200 . Set the parameters of Serial Port 2, and set the baud rate to 9600. Set the interval of timer to 50 milliseconds. Check the data sign of Serial Port 2, and determine whether to receive the data transmitted from sensor. If data is received, carry out cyclic redundancy check, and determine whether there is any transmission error. If there is not any error, further verify whether the sensor address of return data is consistent with that of request data. If yes, it is proved to receive the correct sensor return data, and further process sensor data.

Then, determine whether 3-seconds timer time is up. If yes, send "read sensor" instructions to sensor nodes. If not, return to check whether serial data has been received, and further determine whether correct sensor return data has been received. If not, carry out error processing, and display corresponding sensor faults on screen.

Finally, check whether all sensor data has been read. If yes, transmit all sensor data to DTU through serial port, and further to servers. If not, start 50 milliseconds timer, and continue to read the next sensor.

\section{Information Management System of IoAHT}

Main functions of information management system include as follows: user login; setting station information that user can view according to the users' permission; collecting real-time monitoring data from all the environmental monitoring stations; visualizing the distribution of all the environmental monitoring stations and the real-time environment monitoring data on map; displaying the information of each site including site name, current monitoring image, geographic location information, user name, setup time, current environmental monitoring data and historical monitoring data; retrieving and displaying of historical monitoring data. The user interface of information management system was shown in Fig. 4. 


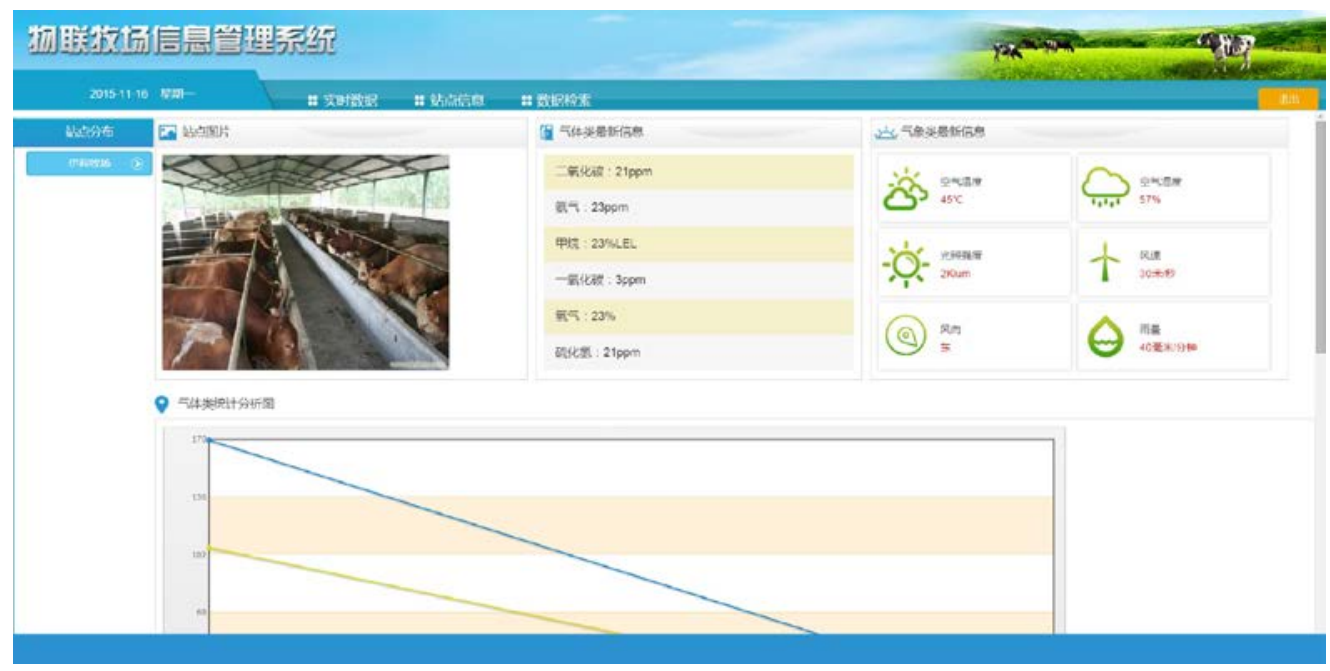

Fig.4. User Interface of Information Management System of IoAHT.

\section{Mobile APP of IoAHT}

Main software functions of mobile APP include system login, verifying user account and password; data acquisition, collecting data from environment monitoring system; system setting, setting server name, IP address and port number. The software has provided a convenience for livestock and poultry breeding management personnel, greatly increased the management efficiency of livestock and poultry breeding, improved the livestock and poultry breeding environment, and maximize the animal welfare. The user interface of mobile APP was shown in Fig. 5.

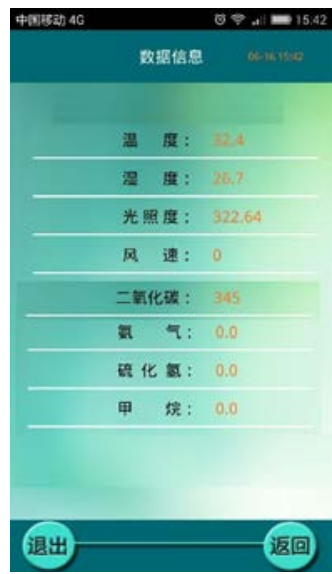

Fig.5. User Interface of Mobile APP

\section{Conclusion}

In this paper, a design scheme of environmental monitoring system based on IoAHT has been presented. The design of environmental monitoring station, information management system, and mobile APP were described in detail. The system can realize real-time acquisition, upload, storage and display of breeding environment parameters. The real time environmental monitoring data can be shown by mobile APP. The system facilitates ranch management personnel to grasp the ranch environment timely, increase the management efficiency, and maximize the animal welfare.

\section{Acknowledgement}

In this paper, the research was sponsored by Beijing Natural Science Foundation Pre-Research Project "Research on Monitoring and Early Warning of Beijing Pork Market Based on Multi-Source Data Fusion” (Grant No.: 9153023) and the National Natural Science Foundation of China (Grant No. 31501229). 


\section{References}

[1] Kong Fantao, New Change of Modern Animal Husbandry in IoAHT [J]. Agricultural Product Market Weekly, 2014,(48):36-39.DOI:10.3969/j.issn.1009-8070.2014.48.014.

[2]Xiong Lu, Zhang Jianhua, Han Shuqing, Advancement and Trend of Internet of Animal Husbandry Things [J]. The Chinese Livestock and Poultry Breeding, 2015,11(11):24-27.DOI:10.3969/j.issn.1673-4556.2015.11.012.

[3] Hao Xiangyang, Wang $\mathrm{Ku}$, Li Moxue, Application of GPRS Wireless Data Transmission in Agricultural Condition Monitoring System [J]. Agricultural Mechanization Research, 2006:186-188. DOI:doi:10.3969/j.issn.1003-188X.2006.06.066.

[4] Zhang Guoyang, Hao Wanjun, Li Ze, et al, Design and Implementation of Agricultural Greenhouse Environment Monitoring System Based on ZigBee and GPRS [J]. Electronic Design Engineering, 2014, 22. DOI:doi:10.3969/j.issn.1674-6236.2014.20.027.

[5] Lei Yang, Making The Corporation of Green Domestic Animals and Poultry to Reduce Agriculture Environmental Pollution [J], Food and Nutrition in China, 2005,(10):55-57.DOI:10.3969/j.issn.1006-9577.2005.10.020.

[6] Yu Fuman, Cai Kezhou, Cheng Yuming, Problems and Countermeasures of Livestock and Poultry Product Quality Safety in China [J]. Food and Nutrition in China, 2011,17(2):10-12.DOI:10.3969/j.issn.1006-9577.2011.02.002.

[7] Ouyang Hongfei, Research on Monitoring and Controlling Technology of Air Environment Quality of Confined Sheep House in Winter in Xinjiang [D]. Xinjiang: Xinjiang Agricultural University, 2008. DOI:10.7666/d.y1256740. 